Supporting information

\title{
The internalization of Garlic-derived Nanovesicles on Liver cells is triggered by interaction with CD98
}

\section{Heliang Song ${ }^{1}$, Brandon S.B. Canup ${ }^{1}$, Vu L. Ngo², Timothy L. Denning ${ }^{2}$, Pallavi Garg ${ }^{2}$, Hamed Laroui ${ }^{1,2^{*}}$}

${ }^{1}$ Department of Chemistry, Center for Diagnostics and Therapeutics (CDT), Georgia State University, Atlanta, GA, USA.

2Department of Biology, Institute for Biomedical Sciences (IBMS), Georgia State University, Atlanta, GA, USA.

* To whom correspondence should be addressed: Hamed Laroui, Department of Chemistry, Center for Diagnostics and Therapeutics (CDT), Georgia State University, Atlanta, GA, 30302, USA;(e-mail: hlaroui@gsu.edu).

\section{Supplementary experimental procedures}

\section{TEM measurement}

A drop of sample (10 $\mu \mathrm{g}$ of protein) was deposited onto the surface of a formvar-coated copper grid, after which $1 \%$ uranyl acetate was added and the sample was drying at room temperature for further imaging.

\section{AFM measurement}

A drop of samples (10 $\mu \mathrm{g}$ of protein) was deposited onto a freshly cleaved mica slide and drying in a cleaning vacuum hood with a cover at room temperature. AFM images were acquired using a SPA 400 AFM instrument (Seiko Instruments Inc., Chiba, Japan).

In-gel digestion and MS experiments

The concentration of the GDVs was calculated by the total protein concentration using the DC assay (BIO-RAD, Hercules, CA). GDVs were incubated with trypsin $(0.25 \%$, Corning) at a mass ratio of $2: 1$ at $37^{\circ} \mathrm{C}$ for $1,2,3,4 \mathrm{~h}$. After digestion, the pellets were collected by ultracentrifugation at $120,000 \times \mathrm{g}$ for 2 hours. The pellet was washed with cold PBS 3 times and resuspended with 1X PBS and labeled as T1, T2, T3, and T4 GDVs respectively for $1 \mathrm{~h}, 2 \mathrm{~h}, 3 \mathrm{~h}$, and $4 \mathrm{~h}$ of enzymatic digestion. 
After ultracentrifugation, GDVs were lysis in RIPA buffer for protein extraction. The gel was run and then stained using Denville protein blue stain. Briefly, the gel was microwaved first with the $250 \mathrm{~mL}$ of $\mathrm{DI}$ water for $3 \mathrm{~min}$ twice, then microwaved with $50 \mathrm{~mL}$ of Denville protein blue stain solution for $2 \mathrm{~min}$, another $2 \mathrm{~min}$ in $250 \mathrm{~mL}$ of DI water.

The in-gel digestion was conducted by the method described before. ${ }^{1}$ Gel spots were picked from the $1 \mathrm{D}$ gel and washed with double distilled water for 3 times. The spots were washing with 400 $\mu \mathrm{l}$ of $25 \mathrm{mM}$ ammonium bicarbonate/methanol for $1 \mathrm{~min}$. The destaining of Coomassie blue was conducted by incubating the gel spots in $400 \mu \mathrm{l}$ of $25 \mathrm{mM}$ ammonium bicarbonate/acetonitrile for 5 min and 100\% acetonitrile for $1 \mathrm{~min}$, respectively. The acetonitrile was removed and gels were dried using speed-vacuum. The gel spots were completely covered with trypsin for $10 \mathrm{~min}$ to saturate them with trypsin. After that, $60 \mu$ of $50 \mathrm{mM}$ ammonium bicarbonate was added and the tubes were placed in an air circulation thermostat and incubate overnight at $37^{\circ} \mathrm{C}$. Then we transferred it to a new microcentrifuge tube and added $60 \mu \mathrm{l}$ of $2.5 \%$ TFA to extra spots for $10 \mathrm{~min}$. The volume of the combination was reduced to $20 \mu$ and submitted to mass spectroscopy analysis (MS/MS).

Nano RP HPLC-MS experiments were performed on an LTQ-Orbitrap Elite mass spectrometer (Thermo Fisher) equipped with EASY-spray source and nano-LC UltiMate 3000 high- performance liquid chromatography system (Thermo Fisher) as described previously. EASY-Spray PepMap C18 Column (75 $\mu \mathrm{m} \times 15 \mathrm{~cm}, 3 \mu \mathrm{m}$, ThermoFisher, US) was used for separation. The separation was achieved with a linear gradient from 3 to $40 \%$ mobile phase B for $80 \mathrm{~min}$ at a flow rate of $300 \mathrm{~nL} / \mathrm{min}$ (mobile phase A: $1.95 \% \mathrm{ACN}, 97.95 \% \mathrm{H} 2 \mathrm{O}, 0.1 \%$ formic acid (FA); mobile phase B: $79.95 \%$ ACN, $19.95 \%$ H2O, $0.1 \%$ FA). LTQ-Orbitrap Elite mass spectrometer was operated in the data-dependent mode. A full-scan survey MS experiment $(\mathrm{m} / \mathrm{z}$ range from 400 to 1600 ; automatic gain control target, $1,000,000$ ions; resolution at $400 \mathrm{~m} / \mathrm{z}, 60,000$; maximum ion accumulation time, $50 \mathrm{~ms}$ ) was acquired by the Orbitrap mass spectrometer, and ten 
most intense ions were fragmented by collision-induced dissociation (CID). The other conditions used were as follows: capillary temperature of $200^{\circ} \mathrm{C}$, collision energy of $35 \mathrm{eV}$.

An Uniprot's garlic database fasta file (3155 items, May 2017) was used for MS/MS spectra matching as reported. ${ }^{2}$ Raw data were analyzed by using Proteome Discoverer 1.4 (ThermoFisher) to search against Uniprot's garlic database (3155 items, May 2017). Searching parameters were used as followed: fixed modification, carbamidomethyl (Cys); variable modifications, deamination (Asn), and oxidation (Met). Trypsin was selected as the enzyme, and two missed cleavages were allowed. The mass tolerance for the precursor ions and the fragment ions was set to $20 \mathrm{ppm}$ and $0.5 \mathrm{Da}$, respectively. A false discovery rate (FDR) of $1 \%$ was applied to all data sets at the peptide level. 
Supplementary experimental results

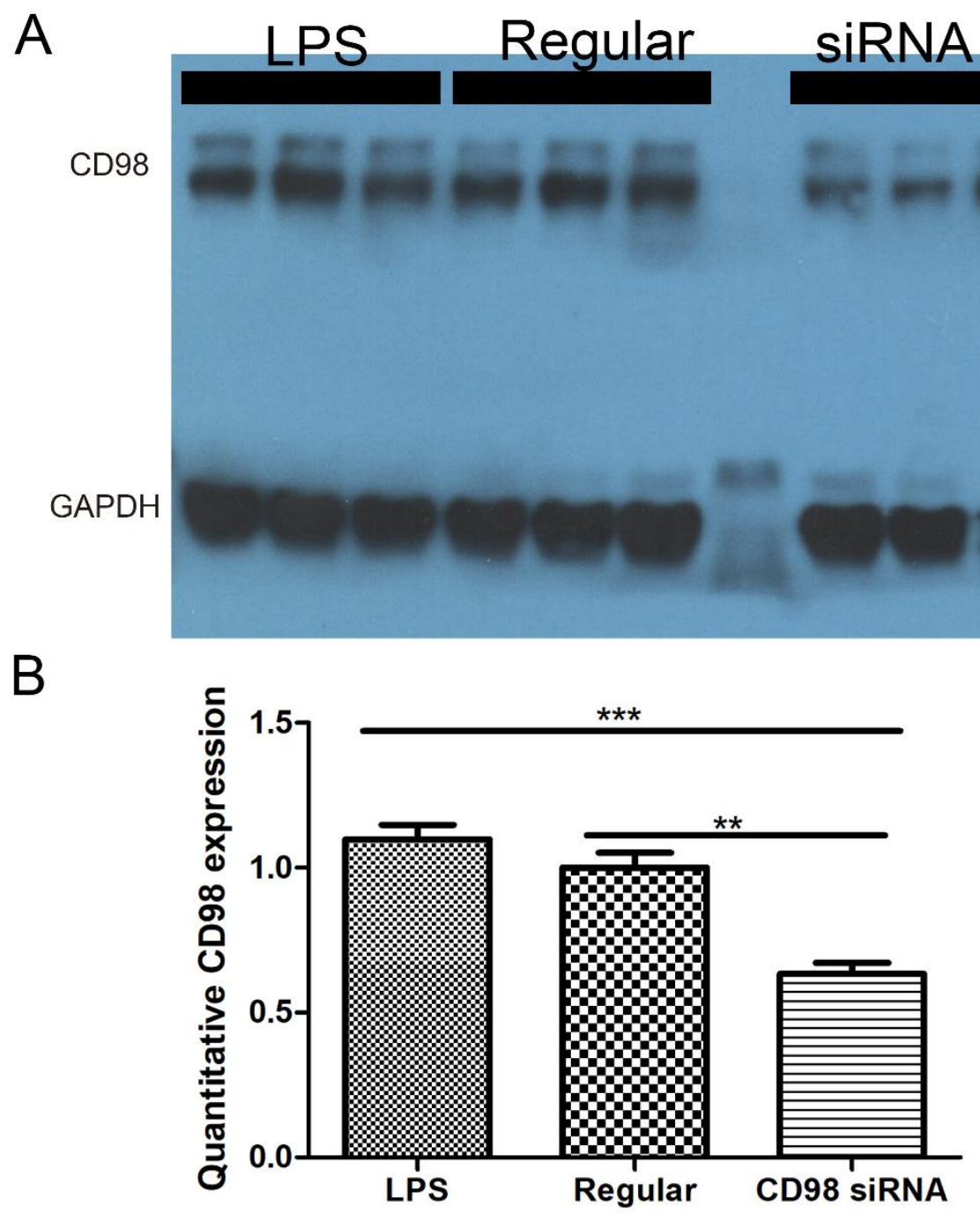

Figure S1. The differential expressions of CD98 were quantified. (A) the expression level of CD98 under three different treatments (LPS, control, CD98 siRNA). (B) the expression amount was quantified. Ttest was used to analyze the significance. 


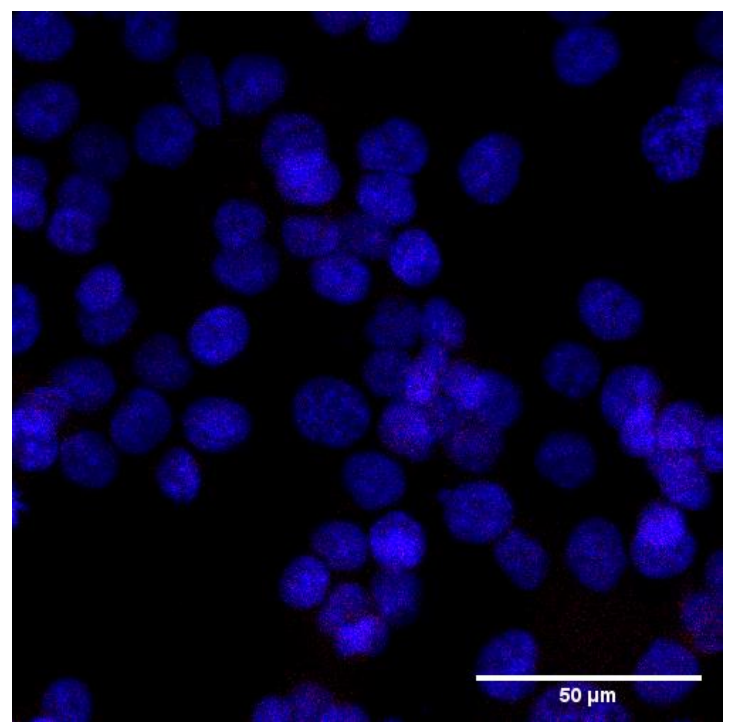

Figure S2. The background of secondary antibody in the staining of CD98 in LPS-treated

HepG2 cells. Same staining procedures with the CD98 staining except that CD98 primary antibody is not applied. The secondary antibody (anti-Rabbit) is tagged with Alexa Fluor 568 (if it is present, it is indicated as red) and the nuclear was stained in blue (DAPI).

\section{Reference}

1. Shevchenko, A.; Tomas, H.; Havli, J.; Olsen, J. V.; Mann, M., In-gel digestion for mass spectrometric characterization of proteins and proteomes. Nat. Protoc. 2006, 1 (6), 2856-2860.

2. Atwood, J. A., 3rd; Sahoo, S. S.; Alvarez-Manilla, G.; Weatherly, D. B.; Kolli, K.; Orlando, R.; York, W. S., Simple modification of a protein database for mass spectral identification of $\mathrm{N}$-linked glycopeptides. Rapid Commun. Mass Spectrom. 2005, 19 (21), 3002-6. 